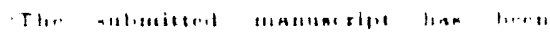

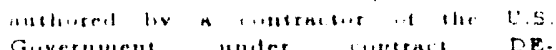
Guverument uater Wutrat DE

ACos.8ton21406). Arcurdingly, the U.S.

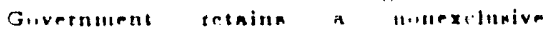

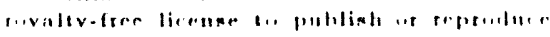

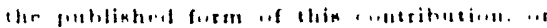

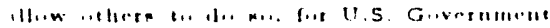
pitrinenen.

\title{
PLASMA TURBULENCE CALCULATIONS ON THE INTEL IPSC/860 (RX) HYPERCUBE*
}

\author{
V. E. Lynch \\ Computing and Telecommunications Division \\ Martin Marietta Energy Systems, Inc. \\ ()ak Ridge, TN 3783i, USA \\ B. A. Carreras, J. B. Drake, I. N. Leboeuf \\ ()ak Ridge National Laboratory \\ ()ak Ridge, TN 3i8:31. USA \\ J. R. Ruiter \\ Albion College \\ Albion. Mll 492'i USA
}

\begin{abstract}
One approach to improving the real-time efficiency of plasma turbulence calculations is to use a parallel algorithm. A serial algorithm used for plasma turbulence calculations was modified to allocate a radial region in each node. In this way, convolutions at a fixed radius are performed in parallel, and communication is limited to boundary values for each radial region. For a semi-implicit numerical scheme (tridiagonal matrix solver), there is a factor of 3 improvement in efficiency with the Intel iPSC/860 machine using 64 prccessors uver a single-processor (ray-II. For block-tridiagonal matrix cases (fully implicit code). a second parallelization takes place. The Fourier components are distributed in nodes. In each node, the block-tridiagonal matrix is inverted for each of the allocated Fourier components. The algorithm for this second case has not yet been optimized.
\end{abstract}

* Research sponsored by the ()ffice of Fusion Energy. U.S. Department of Energy, under contract DE-A(C5-8f()R21 100$)$ with Martin Marietta Energy Systems. Inc. 
NOMENCLATURE

\begin{tabular}{|c|c|}
\hline .4 & $\begin{array}{l}\text { aspect ratio of the forus } \\
\left(\equiv R_{0} / a\right)\end{array}$ \\
\hline$a$ & minor radius of the torus \\
\hline$B_{n}$ & toroidal magnetic field \\
\hline$B_{p}$ & poloidal magnetic field \\
\hline Cind & cooling rate multiplier \\
\hline$E_{\zeta}$ & toroidal electric field \\
\hline$I_{z}$ & coronal equilibrium cooling rate \\
\hline$J_{\zeta}$ & toroidal current density \\
\hline$m$ & poloidal mode number \\
\hline$n$ & density \\
\hline$n$ & toroidal mode number \\
\hline$R_{11}$ & major radius of the torus \\
\hline$r$ & radial coordinate \\
\hline$S$ & Lindquist number \\
\hline$T$ & temperature \\
\hline$t$ & time \\
\hline$U^{\prime}$ & $\begin{array}{l}\text { toroidal component of the } \\
\text { vorticity }\end{array}$ \\
\hline $1^{:}$ & velucity \\
\hline$Z$ & impurity charge \\
\hline Z.eff & $\begin{array}{l}\text { effective impurity charge } \\
=1+Z^{2}\left(n_{z} n_{e}\right)\end{array}$ \\
\hline
\end{tabular}

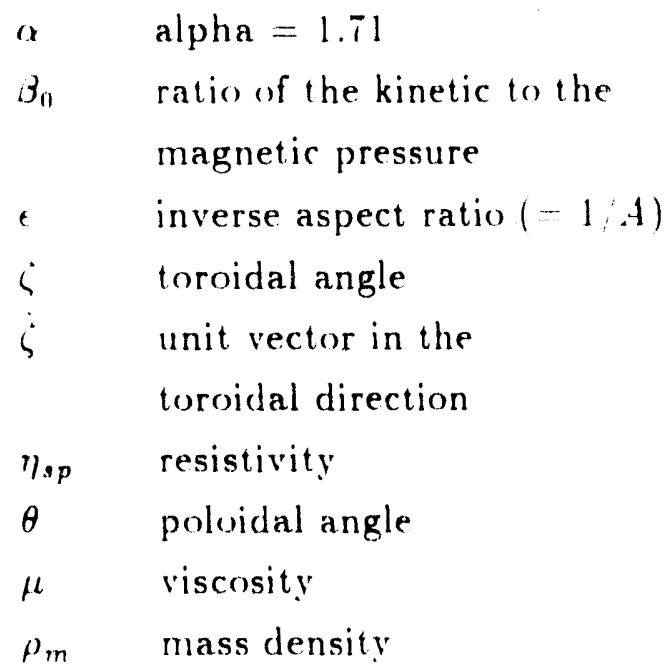

$(-) \quad$ velocity stream function

$\gamma \quad$ transport coefficient

$\Psi$ poloidal magnetic flux

$\omega$ diamagnetic frequency

$\omega_{c} \quad$ ion cyclotron frequency

Superscripts and subscripts

eff electron
eq eqfective
$i \quad$ ion
$=\quad$ impurity species
Parallel to the magnetic field
perpendicular to the magnetic

field
perturbed quantities




\section{Introduction}

The study of plasma turbulence and the concomitant transport is a challenging problem. The fluid approach to plasma dynamics makes tractable some problems that are, at present, ton complex in kinetic theory. This approach assumes that the plasma is an electrically conducting fluid, which can be confined inside a magnetic field because it tends to flow mostly along field lines but not across them.

For pure magnetohydrodynamic (NHD) problems in the large-aspect-ratio limit, the basic equations can be reduced to two partial differential equations. ${ }^{1}$ These equations can be solved using a semi-implicit scheme that requires a tridiagonal matrix solver. ${ }^{2}$ This model has been used to study tearing mode turbulence with application to tokamak disruptions ${ }^{3}$ and dynamo effects in reversed-field pinch configurations. ${ }^{4}$

In general, transport studies in toroidal configurations require one to go beyond the simple MHD model. The effects of temperature, density, etc., must be included. As a consequence, we must increase the number of partial differential equations to be solved. In the case oi edge plasma turbulence. we have used a six-equation model, treating the linear terms of these equations implicitly. These require the inversion of block-tridiagonal matrices. ${ }^{5}$

As is typical of turbulence studies, high resolution is needed to resolve all scale lengths in the problem. This requires large amounts of memory. To study the development of steady-state turbulence, the plasma evolution must be followed for many time steps. Therefore, these calculations require large amounts of computing time, and in real time, they extend over periods of many months. ${ }^{6}$

Une approach to improving the real-time efficiency of plasma turbulence calculations is to use a parallel algorithm that we first tested on the 64-node Intal iPSC hypercube. ${ }^{7}$ The semi-implicit algorithm described in Reference 2 was modified to allocate a radial region in each node of the hypercube. In this way, the convolutions at a fixed radius were performed in parallel, and communication between nodes was limited to the boundary values for each radial region. The memory configuration used in this implementation was a ring. Here, we compare the resuits of these calculations with the use of a shared memory machine such as the C'ray-lI.

The fully implicit algorithm described in Reference 5 has also been adapted to the hypercube. For the calculation of the convolutions, we use the same approach as for the semi-implicit scheme. However, in this case, a second parallelization in which the Fourier components are distributed in nodes is required. This algorithm has not yet been iptimized.

The rest of the paper is organized as follows. In Section 2, the two sets of equations used in the present studies are described, and the respective algorithms are briefly summarized. The implementation of these schemes in the hypercube is discussed in Section 3. The iming results for different numbers of processors are presented in Section $t$. 


\section{Equations and Numerical Scheme}

In the case of toroidal confinement devices (tokamak and stellarators), the magnetic field line topology is a three-dimensional torus. These magnetic configurations are created by a toroidal field, $B_{0}$, the long way around the torus and a poloidal magnetic field, $B_{p}$, the short way around the torus. In equilibrium, the toroidal field is larger than the poloidal field, and they obey the ordering $B_{0} / B_{p}=A>1$, where $A \equiv R_{0} / a$ is the aspect ratio of the torus. In a large-aspect-ratio approximation $(A \gg 1)$, the MHD equations can be reduced to

$$
\begin{aligned}
& \frac{\partial \bar{\Psi}}{\partial t}=-\frac{R_{0}}{B_{0}} \Gamma_{\|} \tilde{\Phi}+\eta_{s p} J_{\zeta} \cdots E_{\zeta}, \\
& \frac{\partial \bar{U}}{\partial t}=-\bar{\Gamma} \cdot \Gamma \bar{U}-S^{2} \Gamma_{\|} J_{\zeta}+\mu \Gamma_{\perp}^{2} \bar{U}
\end{aligned}
$$

Here $\Psi$ is the poloidal magnetic flux, $\vec{B}=1 / R \Gamma \tilde{\Psi} \cdot \dot{\zeta}+B_{0} \dot{\zeta}, \dot{\zeta}$ is the unit vector in the toroidal direction. $\dot{l} \equiv \zeta \cdot\left(\Gamma, 1^{\circ}\right)$ is the toroidal component of the vorticity, $\Phi$ is the velocity stream function, and $\vec{r} \equiv \Gamma \bar{\phi} \cdot \zeta$ is the velocity. Parameters in these equations are the resistivity $\eta_{s p}$, the major radius of the torus $R_{0}$, the minor radius of the torus $a$, the riscosity $\mu$, and the mass density $\rho_{m}$.

The toroidal current density, $J_{\zeta}$, in terms of the poloidal flux is given by

$$
J_{\zeta}=\frac{1}{R_{0} \mu_{0}} \Gamma_{\perp}^{2} \Psi
$$

The toroidal current is driven by a constant electric field $E_{\zeta}$.

In describing the plasma turbulence. it is necessary to include the effect of density and temperature gradients, parallel flows, etc. Therefore, a more complete set of equations is required. In particular. to study plasma edge turbulence in tokamaks, we have used the following set of equations:

$$
\begin{aligned}
& \frac{\partial \bar{\Psi}}{\partial t}=\frac{R_{0}}{B_{n}} \Gamma_{: !} \tilde{\Phi}+\eta_{s p} Z_{e f f} J_{\zeta} \quad E_{\zeta}-S \omega_{e}^{\prime} \frac{T_{e}}{n_{e}} \Gamma_{\|} n_{e}-S \omega_{e}^{\prime}(1+\alpha) \Gamma_{\|} T_{e}, \\
& \frac{\partial \dot{U}}{\partial t}=-\dot{1} \cdot \Gamma \dot{r}-S^{2} \Gamma_{\mu} J_{\zeta}-\frac{1}{2} \vec{\zeta} \cdot\left(\Gamma n_{e} \cdot \Gamma I_{\perp}^{2}\right)+\mu \Gamma_{\perp}^{2} \dot{U},
\end{aligned}
$$

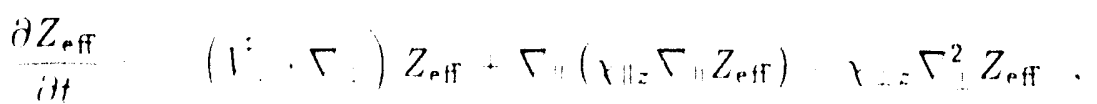

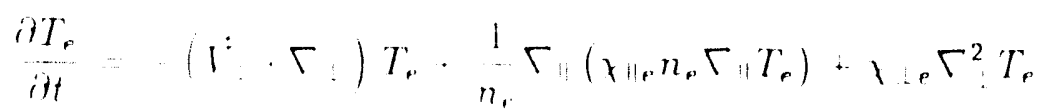

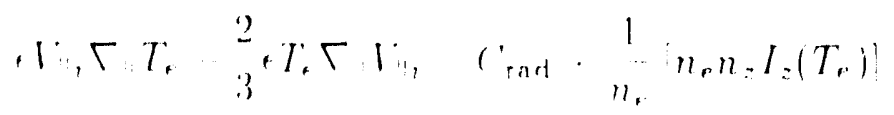

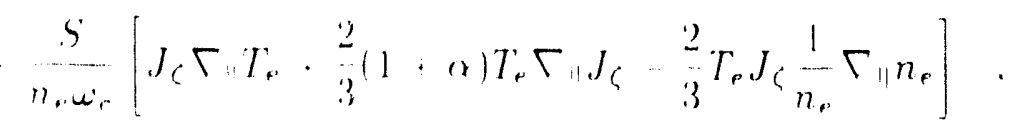




$$
\begin{aligned}
\frac{\partial n_{e}}{\partial t}= & -\left(\vec{V}_{\perp} \cdot \Gamma_{\perp}\right) n_{e}-\epsilon V_{\| i} \Gamma_{\|} n_{e}-\epsilon n_{e} \Gamma_{\|} V_{\| i}+\frac{S}{\omega_{c}} \Gamma_{\|} J_{\zeta}+\chi_{\perp} \Gamma_{\perp}^{2} n_{e}, \\
\frac{\partial V_{\| i}}{\partial t}=- & \left(\vec{V}_{\perp} \cdot \Gamma_{\perp}\right) \Gamma_{\| i}-\epsilon V_{\| i} \Gamma_{\|} V_{\|_{i}}+\frac{1}{n_{e}} \Gamma_{\|}\left(n_{e} \mu_{\| i} \Gamma_{\|} V_{\| i}\right)+\mu_{\perp i} \Gamma_{\perp}^{2} V_{\|_{i}} \\
& -\frac{\beta_{0 e} S^{2}}{2 \epsilon} \frac{1}{n_{e}}\left[\Gamma_{\|}\left(n_{e} T_{e}\right)+\Gamma_{\|}\left(n_{i} T_{e}^{\text {eq }}\right)+\Gamma_{\|}\left(n_{z} T_{e}^{\text {eq }}\right)\right] .
\end{aligned}
$$

This set of equations is an extension of the previous one for two fluids plus an impurity species. Here, $n_{e}$ and $n_{z}$ are the electron and impurity density, $T_{e}$ is the electron temperature, $V_{\|_{i}}$ is the component of the ion velocity parallel to the magnetic field and $Z_{\text {eff }}$ is related to the impurity charge, $Z$, by

$$
Z_{\text {eff }}=1+Z^{2} \frac{n_{z}}{n_{e}}
$$

The function $I_{z}\left(T_{e}\right)$ is the cooling rate caused by radiation as given by the coronal equilibrium model. The $C_{\text {rad }}$ multiplier is used to adjust this cooling rate to the experimental measurement. The ion density is obtained through the quasi-neutrality relation $n_{e}=n_{i}+Z n_{z}$. The subindexes $\|$ and $\perp$ indicate parallel and perpendicular to the magnetic field. Important dimensionless parameters in these equations are the Lindquist number $S$, the ratio of the kinetic to the magnetic pressure $\beta_{0}$, and $\varepsilon=1 / \mathrm{A}$. Parallel and cross-field transport coefficients for the electrons and impurities are decigned by $\chi$ with proper subindexes. The coefficient $\alpha=1.71$.

To numerically integrate these equations, all fields are expressed as the sum of an equilibrium quantity and a perturbed quantity,

$$
f(r, \theta, \dot{\zeta})=f_{0}(r)+\dot{f}(r, \theta, \dot{\zeta}) .
$$

where $r$ is the radial coordinate and $\theta$ and $\zeta$ are the poloidal and toroidal angles. The perturbation part of all fields is written as an expansion in sines and cosines,

$$
\bar{f}(r, e . \dot{\zeta})=\sum_{m n} f_{m n}^{c}(r) \cos (m \theta+n \zeta)+f_{m n}^{s}(r) \sin (m \theta+n \dot{\zeta})
$$

The boundary conditions are that the radial components of the magnetic field and velocity are zero at the conducting wall, $r=a$, and so is the pressure. That is, for all fields.

$$
\tilde{f}(a, \theta, \zeta)=0
$$

Fin complete the numerical representation of the fields. we use finite differences in $r$. Each scalar field is written as a Fourier expansion, Eq. (10). The components $f_{m n}^{c}(r)$ and $f_{m n}^{s}(r)$ are functions of $r$ represented on a finite-difference grid. First and second radial derivatives are calculated with three-point finite-difference formulas that allow unequal spacing. This allows us to concentrate the grid where the steepest gradients are located. lerivatives in $\theta$ and $\zeta$ are performed analytically. All quantities are stored in spectral form and are never transformed to a finite-difference grid in $\theta$ and $\zeta$. The convolutions 
are performed analytically, rather than using fast Fourier transforms. For plasma edge turbulence, the radial grid is concentrated in the region where driving terms are effective. lor high-resolution calculations, this allows us to use a radial grid spacing $\Delta r \approx 10^{-3} a$. To obtain the desired resolution in poloidal and toroidal angles, the number of Fourier components used is about 1000 .

These nonlinear equations are solved as an initial value problem. The numerical scheme used in solving Eqs. (1) and (2) is implemented in the code RSF and explained in detait in Reference 2. It is a two-step algorithm. In the first half step, Eq. (2) is advanced explicitly while Eq. (1) treats the linear terms as implicit. The second half of the time step is explicit and such that the overall scheme is $\Delta t^{2}$ accurate. Each time step requires the inversion of three tridiagonal matrices for each Fourier component, twice to find $\Phi$ in terms of $U$ and once for the implicit advancement of Eq. (1).

The numerical scheme presently used ${ }^{5}$ in solving Eqs. (3)-(8) is implemented in the code KITE and treats all linear terms in the perturbation implicitly; the nonlinear terms are explicit and accurate to first order in time. This requires the inversion of a blocktridiagonal matrix for each Fourier component.

These calculations have been routinely performed using the Cray-Il computer available at the National Energy Research Supercomputer Center. High-resolution turbulence calculations require several hundred hours of Cray-II (YPI. 


\section{Numerical Implementation in the Hypercube}

To improve the real-time efficiency of plasma turbulence calculations, the algorithm described in Reference 2 was modified to allocate a radial region in each node of the hypercube. In this way, the convolutions at a fixed radius were performed in parallel, and communication between nodes was limited to the boundary values for each radial region. The memory configuration used in this implementation was a ring.

The tridiagonal matrix solver is based on the standard forward elimination and back substitution method. Its parallelization requires communication betweer the nodes. The cuefficients of each tridiagonal system associated with a given mode are divided among the processors. The formation of each system can thus be accomplished with perfect parallelism. The solution procedure, however, achieves only partial parallelism. Once the systems are formed for all the modes in each processor, the solution method proceeds as follows. The first processor eliminates the portion of the system it owns for the first mode. It then sends the three boundary values to the next processor, so that the latter can begin the elimination on the first mode. After the send, the first processor can begin elimination if the second mode while the second processor works on the first mode. In this fashion, a pipeline is established, with the eventual filling of the pipe and synchronization of computation and communication. Thus, the elimination algorithm achieves some parallelism but incurs a pipeline startup cost. The back substitution algorithm works similarly.

For the more complex problems, Eqs. (3)-(8), a fully implicit scheme for the linear terms is used. In this case. the matrix is block tridiagonal. For block-tridiagonal matrix cases. the matrix solver numerical scheme has been changed to increase the level of parallelization. To accomplish this, after the convolutions have been evaluated, the memory allocation is redistributed. Several modes are allocated to each processor for all radial grid points. In each processor, the block-tridiagonal matrix is inverted for each of the allocated modes. Before the inversion. each node must have the corresponding right-hand side for all the radial grid points. Each processor sends a vector to all the other nodes in an embedded ring. On return, that processor contains a right-hand side filled with information from all other nodes. After the inversion, the solution must be returned to all the appropriate processors for each radial region. The actual inversion involves no communication between prucessors. The inversion is computed with the block-tridiagonal linear system solver, minimal storage version. BTMS. ${ }^{8}$ The blocks of the matrix and the corresponding blocks if the right-hand side vector are obtained, one block-row at a time, from a user-supplied routine. BTMS uses block Gauss elimination. Partial pivoting is done within block-rows unly. The matrix elements are computed as they are needed with B'TMS so the matrix need not be in the memory of the norle. BTMS was modified to be a parallel multiple independent) block-tridiagunal solver by J. B. Drake and will be documented elsewhere." 


\section{Results}

Calculations are being done on the Intel iPSC/860 (RX) hypercube which was delivered to Oak Ridge National Laboratory on January 2, 1990. ${ }^{10}$ This machine has 128 processors and uses the $i 860$ chip with a clock rate of $40 \mathrm{MHz}$. The peak aggregate gigaflops is 7.0 for double precision, 10 for single precision, and the aggregate memory is $1048 \mathrm{MB}$. It is a distributed-memory, MIMD machine whose processors are synchronized through message passing. In the previous studies, it was shown ${ }^{7}$ that it was possible to use 16 nodes to achieve a. speed-up factor of 4 to 5 . However, with the iPSC/2 it was possible to perform only sample calculations, but no production computations. Present results, before uiuch optimization of the code has been done, show that the number of steps for a given amount of CPU time is 3 times better for the RX machine using 64 processors than for a singleprocessor ('ray-II (for 300 modes and 129 radial grid points). The results of the comparison of the two machines are shown in Fig. 1. In this figure, we have separated the contributions to the time step of the convolutions and of the tridiagonal matrix inversion. Increasing the number of processors decreases the time per step for the convolutions. Since the number of grid points (129) and the number of Fourier components (300) are kept constant, the efficiency improvement with a number of processors levels off when the number of grid points for each node is less than 5 . The time invested in the tridiagonal inversion per step increases with the number of processors because of the increased communication. However, its contribution to the overall time per step remains small. When the number of Fourier components is increased for a fixed number of processors in the RX machine, its efficiency improves over that of the Cray. This is shown in Fig. 2.

Tests running the parallel BTMS solver with 147 matrices containing $3807 \times 7$ blocks indicate that the solver uses the least time for these matrices with 32 processor nodes. These tests did not include the time needed to calculate the matrix elements. As shown in Fig. 3, the time decreased in going from 2 processors to 32 processurs. This decrease occurs because the time is dominated by the calculations in the solver and little communication is needed since there are few nodes. With mie than 32 processors, so few linear systems are being solved per processor and so much communication is required to pass the right-hand sides and answers to the processors that the time is dominated by the communication between processors and increases as more processors are used for the inversion of the matrix. One should note that these tests were carried out with problem sizes far reduced from those needed for production runs. As the size of the problem is made more and more realistic, performance improves steadily when more and more processors are used.

Timing for the KITE code differs from that for the RSF code in that the blocktridiagonal inversion is more expensive to compute than the convolutions. The code timings shown in Fig. t are for 1 t 7 right-hand sides and matrices containing $4 i T$. $T$ blocks. The time for the block-tridiagonal inversion includes the time to calculate the matrix elements, the communication between processors. and the time for the actual inversion. The time does decrease as the numbers of processors increases from 2 1016 and then increases with 32 processors like the lest of in solver in fig. 3. but much needs to be done to make the solver less time-consuming. ('omputing the matrices initially and then reading them from 
the $1 / O$ nodes in the hypercube would reduce the CPU time needed for the solver. Also, other methods of making the solver parallel need to be tested. In particular, a pipeline approach like the one used in the tridiagonal solver of RSF might be less time-consuming. With that approach, communication could be overlapped with computations.

In conclusion, the parallel algorithm offers a possibility of substantially reducing the real time spent in plasma turbulence calculations. At present, the software we have used has not been fully optimized to the hypercube machine. Much more work is required on the present algorithms before the plasma turbulence codes can be used for a routine production mode.

\section{DISCLAIMER}

\footnotetext{
This report was prepared as an account of work sponsored by an agency of the United States Givernment. Neither the I/nited States Government nor any agency thereof, nor any of their eniployees, makes any warranty, express or implied, or assumes any legal liability or responsibility for the accuracy, completeness, or usefulness of any information, apparatus, product, or process disclosed. or represents that its use would not infringe privately owned rights. Reference herein to any specific commercial product, process, or service by trade name, trademark, manufacturer, or otherwise does not necessarily constitute or imply its endorsement, recommendation, or favoring by the United States Government or any agency thereof. The views and opinions of authors expressed herein do not netessarily state or reflect those of the United States Government or any agency thereof.
} 


\section{Acknowledgments}

We would like to thank Drs. John Sheffield, R. A. Dory, and R. C. Ward for their support and encouragement in carrying out this project. 


\section{References}

1. H. R. Strauss, "Nonlinear, three-dimensional magnetohydrodynamics of noncircular tokamaks," Phys. Fluids 19, 134 (1976).

2. H. R. Hicks, B. A. Carreras, J. A. Holmes, D. K. Lee, and B. V. Waddell, "3D nonlinear calculations of resistive tearing modes," J. Comput. Phys. 44, 46 (1981).

3. B. A. Carreras, H. R. Hicks, J. A. Holmes, and B. V. Waddell, "Nonlinear coupling of tearing modes with self-consistent resistivity evolution in tokamaks," Phys. Fluids 23, $1811(1980)$.

4. J. A. Holmes, B. A. Carreras, P. H. Diamond, and V. E. Lynch, "Nonlinear dynamics of tearing modes in the reversed field pinch," Phys. Fluids 31, 1166 (1988).

5. L. Garcia, H. R. Hicks, B. A. Carreras, L. A. Charlton, and J. A. Holmes, "3D nonlinear MHD calculations using implicit and explicit time integration schemes," J. Comput. Physs. 65, 253 (1986).

6. B. A. Carreras, N. Dominguez, J. B. Drake, J.-N. Leboeuf, L. A. Charlton, J. A. Holmes, D. K. Lee, V. E. Lynch, and L. Garcia, "Plasma turbulence calculations on supercomputers," Int. J. of Supercomputer Applications 4, 97 (1990).

7. J. B. Drake, B. F. Lawkins, B. A. Carreras, H. R. Hicks, and V. E. Lynch, "Implementation of a 3-D Nonlinear MHD Calculation on the Intel Hypercube," Report ORNL-6335, 1987.

8. A. C. Hindmarsh, "Solution of Block-Tridiagonal Systems of Algebraic Equations," Report UCID-30150 Lawrence Livermore Laboratory, Livermore, California, 1977.

9. J. B. Drake, private communication.

10. M. T. Heath, G. A. Geist, and J. B. Drake, "Early Experience with Intel iFSC/860 at Oak Ridge National Laboratory," Report ORNL/TM-11655, Oak Ridge National Laboratory, Oak Ridge, 1990. 


\section{Figure Captions}

FIG. 1. Comparison of the performance of the Cray-Il and the Intel iPSC/860 for the RSF code, with the contributions to the time step of the convolutions and of the tridiagonal matrix inversion separated. These timings are for 129 grid points, 300 Fourier components, and 10 time steps.

FIG. 2. Comparison of the performance of the Cray-II and 32 processors of the Intel iPSC/ 860 for the RSF code, for 129 grid points with an increasing number of Fourier components.

FIG. 3. Timings of the parallel block-tridiagonal solver for 147 parallel solves of matrices containing $3807 \times 7$ blocks.

FIG. 4. Timings of the KITE code for 147 modes and 97 radial grid points with the contributions to the time step of the convolutions and of the block-tridiagonal matrix inversion separated. 
ORNL.-DWG 90M-3147 FED

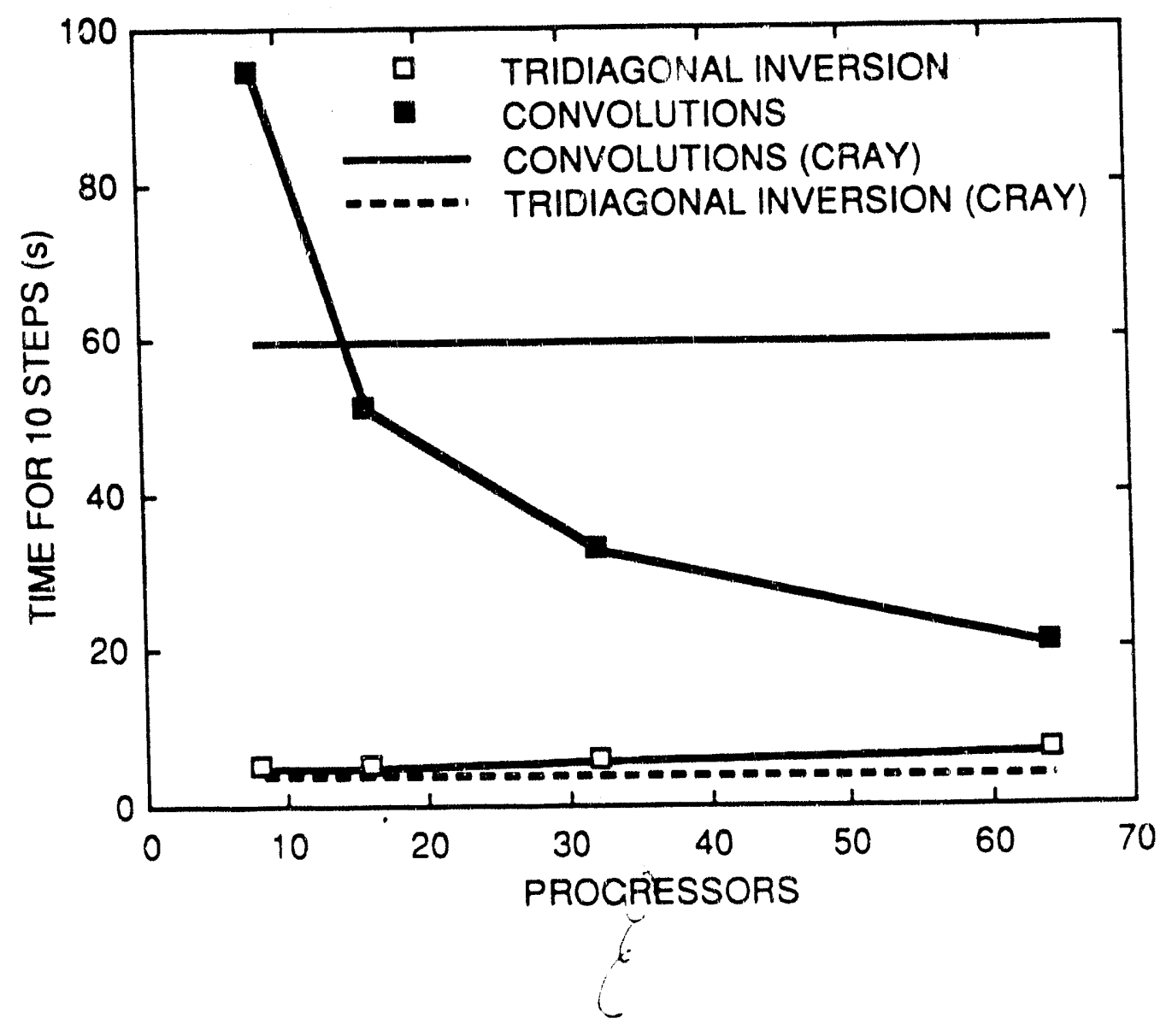




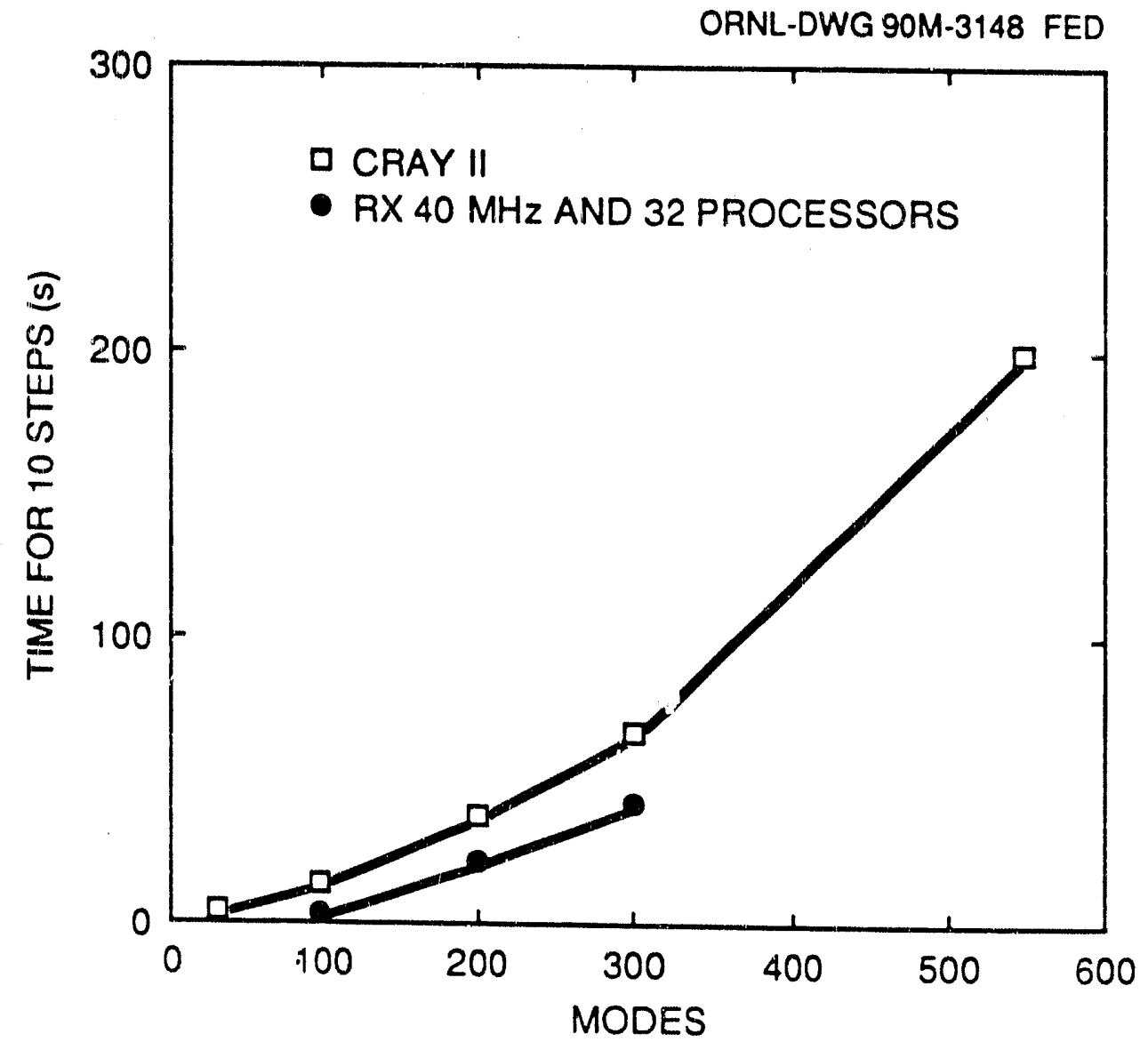




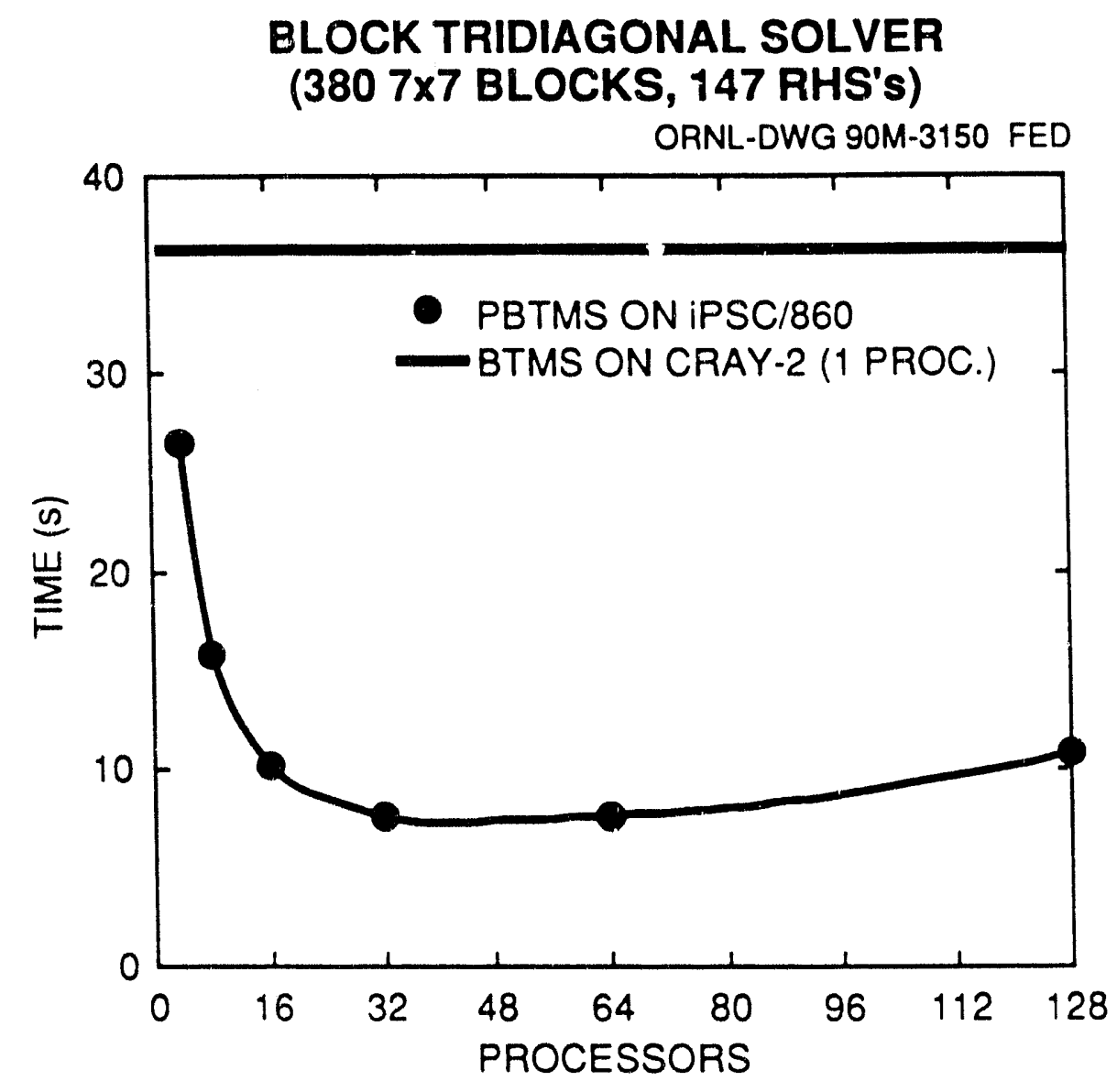


ORNL-DWG 9OM-3149 FED

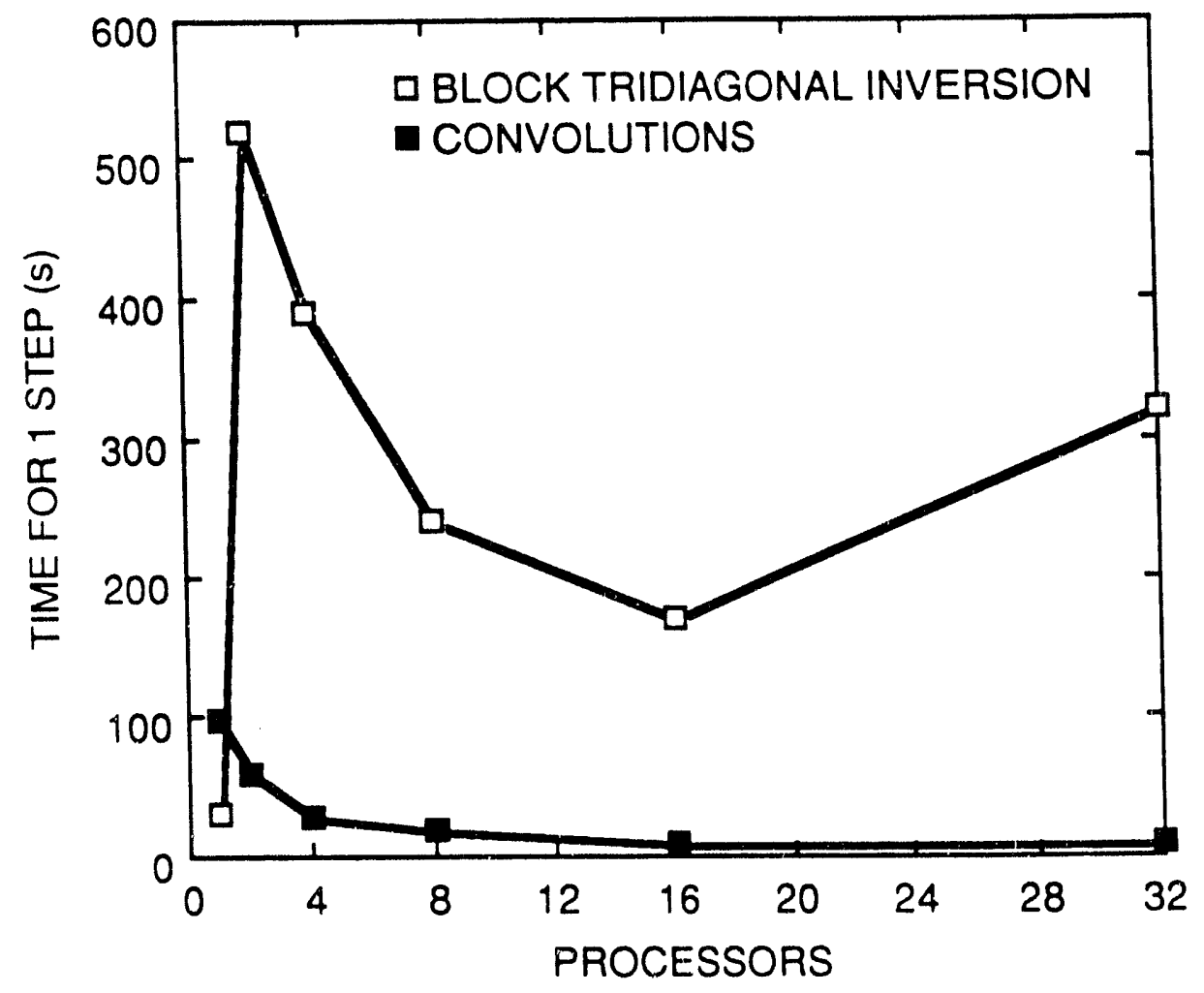



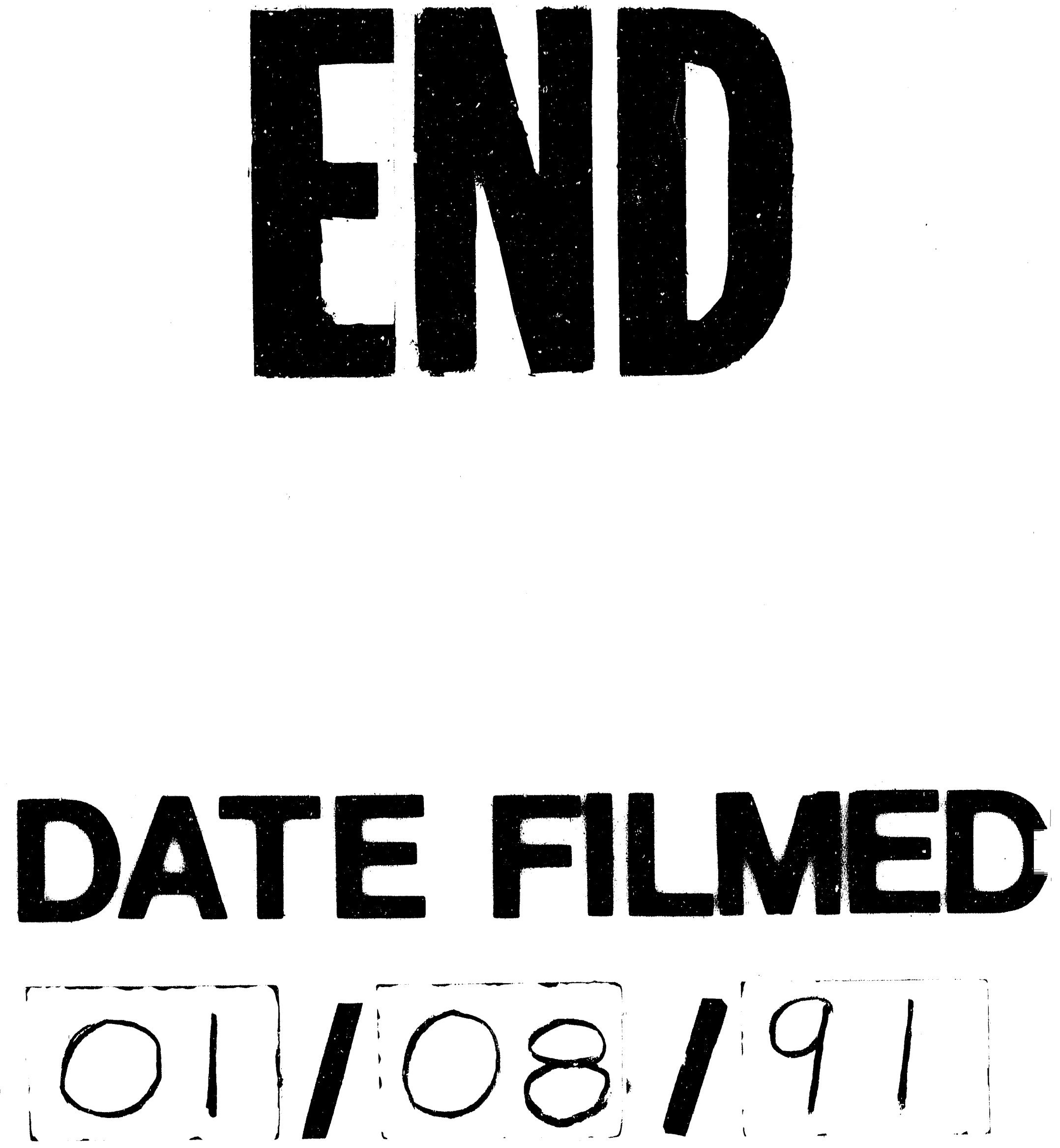
